ARTICLE

DOI: $10.1038 / s 41467-018-06740-x$

\title{
Enhanced mRNA FISH with compact quantum dots
}

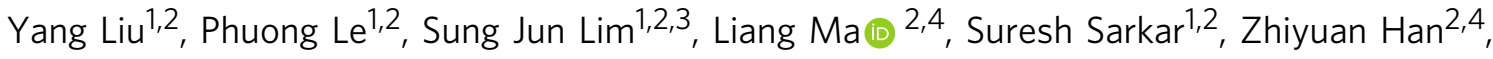 \\ Stephen J. Murphy (10 ${ }^{5}$, Farhad Kosari ${ }^{5}$, George Vasmatzis ${ }^{5}$, John C. Cheville ${ }^{5,6}$ \& Andrew M. Smith (1) 1,2,4,7
}

Fluorescence in situ hybridization (FISH) is the primary technology used to image and count mRNA in single cells, but applications of the technique are limited by photophysical shortcomings of organic dyes. Inorganic quantum dots (QDs) can overcome these problems but years of development have not yielded viable QD-FISH probes. Here we report that macromolecular size thresholds limit mRNA labeling in cells, and that a new generation of compact QDs produces accurate mRNA counts. Compared with dyes, compact QD probes provide exceptional photostability and more robust transcript quantification due to enhanced brightness. New spectrally engineered QDs also allow quantification of multiple distinct mRNA transcripts at the single-molecule level in individual cells. We expect that QD-FISH will particularly benefit high-resolution gene expression studies in three dimensional biological specimens for which quantification and multiplexing are major challenges.

\footnotetext{
${ }^{1}$ Department of Bioengineering, University of Illinois at Urbana-Champaign, Urbana, IL 61801, USA. ${ }^{2}$ Micro and Nanotechnology Laboratory, University of Illinois at Urbana-Champaign, Urbana, IL 61801, USA. ${ }^{3}$ Intelligent Devices and Systems Research Group, DGIST, Hyeonpung, Daegu 42988, Republic of Korea. ${ }^{4}$ Department of Materials Science and Engineering, University of Illinois at Urbana-Champaign, Urbana, IL 61801, USA. ${ }^{5}$ Biomarker Discovery Program, Center of Individualized Medicine, Mayo Clinic, Rochester, MN 55905, USA. ${ }^{6}$ Department of Laboratory Medicine and Pathology, Mayo Clinic, Rochester, MN 55905, USA. ${ }^{7}$ Carle Illinois College of Medicine, Urbana, IL 61801, USA. Correspondence and requests for materials should be addressed to A.M.S. (email: smi@illinois.edu)
} 
$\mathrm{F}$ or a half century, in situ hybridization (ISH) has been used to count, localize, and characterize individual nucleic acids within cells and tissues ${ }^{1,2}$. Originally developed with radioisotopic labels, the advancement of fluorescence ISH (FISH) ${ }^{3}$ led to broad adoption in cytogenetics and clinical diagnostics ${ }^{4}$, today comprising a multi-billion USD industry. Over the past 20 years, FISH has extended to single-RNA analysis ${ }^{5}$, becoming a standardized method for measuring gene expression at the single-cell level, and enabling the discovery of regulatory mechanisms of transcription and translation driven by subcellular localization ${ }^{6,7}$. However signals from organic dye labels used in FISH rapidly deteriorate during photoexcitation, particularly when imaging in three dimensions and under high photon flux needed for superresolution ${ }^{8}$. In addition, the technique is limited to simultaneous analysis of $\sim 3$ RNA targets due to dye emission spectra overlap, unlike high-throughput ex situ techniques like singe-cell whole transcriptome sequencing that simultaneously probe thousands of transcripts from lysed cell extracts. Creative approaches have increased RNA FISH throughput using repeated cycles of labeling, imaging, and label depletion ${ }^{9}$, but the methodologies are laborious and challenging to adopt for non-specialists.

It is widely anticipated that in situ techniques requiring stable, multiplexed probes will substitute dyes with nanocrystalline quantum dots (QDs) due to their extremely stable and intense emission and vastly expanded multiplexing capabilities deriving from narrow emission bands tunable across the ultraviolet, visible, and infrared spectra ${ }^{10}$. But despite concerted efforts, considerable industry investment, and broad use in solution-based assays, QDs have not been widely used in FISH protocols. Presumably this is due to inaccurate labeling resulting from the large sizes $(15-35 \mathrm{~nm})$ of commercially available probes ${ }^{11,12}$, which cannot transport into crowded macromolecular environments of fixed cells to densely label targets. To determine the conditions under which QDs can be applied for accurate counting of mRNA transcripts, rigorous quantification must be applied using wellcontrolled cellular expression systems together with direct comparisons to standardized analytical techniques.

Here we confirm that critical thresholds for cytoplasmic sieving limit RNA FISH and that a new generation of compact and stable QDs can overcome steric hindrance problems to match labeling accuracies of organic dyes. We show that QD-FISH provides improved signal stability, improved fidelity of molecular counting, and the capacity for multiplexed RNA quantification at the single-molecule level.

\section{Results}

Impact of QD size on mRNA labeling. We generated a series of QDs coated with multidentate polymers that allow the total hydrodynamic diameter of the probe to be as small as $\sim 7 \mathrm{~nm}$. These products are stable as off-the-shelf materials for years and are azide-functional for facile conjugation to proteins and nucleic acids through high-precision click-chemistry ${ }^{13}$. Fig. 1a shows representative FISH images of HeLa cells stained for transcripts of glyceraldehyde-3-phosphate dehydrogenase (GAPDH), comparing $\sim 1 \mathrm{~nm}$ organic dyes or QDs with compact $(13.3 \mathrm{~nm})$ or large $(17.4 \mathrm{~nm})$ hydrodynamic diameters, all using the same oligonucleotide probe sequences based on the Raj et al. multiple labeling method $^{8}$. Similar single-molecule counts are observed for dyes (425) and compact QDs (487), whereas counts for large QDs (75) are much lower. RNA counts per cell for a range of probes are quantified as scatter plots in Fig. 1b. We synthesized these QDs as $\mathrm{Hg}_{x} \mathrm{Cd}_{1-x} \mathrm{Se} / \mathrm{Cd}_{y} \mathrm{Zn}_{1-y} \mathrm{~S}$ core/shell structures with a wide range of diameters of 3.3, 5.7, and $8.7 \mathrm{~nm}$ (Fig. 1c), all with emission in the red spectrum (Supplementary Fig. 1), tuned by the core alloy composition parameter $x^{14}$. After polymer coating, the hydrodynamic diameters of the respective aqueous QDs were 9.2 $\mathrm{nm}\left(\mathrm{QD}_{9.2}\right), 13.3 \mathrm{~nm}\left(\mathrm{QD}_{13.3}\right)$, and $17.4 \mathrm{~nm}\left(\mathrm{QD}_{17.4}\right)$ measured by protein-calibrated gel permeation chromatography (GPC, Fig. 1c). Both $\mathrm{QD}_{9.2}$ and $\mathrm{QD}_{13.3}$ yielded $G A P D H$ mRNA counts that were similar to those of dyes $(p>0.05$; Student's $t$-test), whereas counts using $\mathrm{QD}_{17.4}$ labels were significantly lower $(p<$ $0.001)$. An alternative large $Q D$ variant from a commercial vendor $\left(\mathrm{QD}_{\text {com }}\right)$ with dissimilar surface chemistry (PEG-coated amphiphilic polymers) likewise under-labeled RNA targets $(p<$ $0.001)$.

Presumably the majority of GAPDH transcripts are located in crowded cytosolic regions inaccessible to QDs larger than 13.3 nm. We specifically chose $G A P D H$ as a target due to high expression with distribution throughout the heterogeneous cytoplasm. We observed that the transcripts that could be labeled by $\mathrm{QD}_{17.4}$ were also bound to fewer fluorophores compared with $\mathrm{QD}_{13.3}$ and dyes. Fig. 1d shows fluorescence intensities per labeled RNA, compared with single fluorophores, showing that RNAs were labeled with a mean of 8.0 dyes, $10 \mathrm{QD}_{13.3}$, and $2.3 \mathrm{QD}_{17.4}$ (Supplementary Table 3). The low labeling density for $\mathrm{QD}_{17.4}$ shows that steric hindrance is limiting even for the most accessible RNAs, consistent with reports demonstrating substantially greater obstruction of cytosolic diffusion for $\sim 16 \mathrm{~nm}$ pentamers of green fluorescence protein (GFP) than $\sim 11 \mathrm{~nm}$ GFP trimers ${ }^{15}$. For the ensuing work below, we exclusively use $\mathrm{QD}_{13.3}$.

Photostability comparisons. Photostability is substantially improved for QD-FISH compared with Dye-FISH. Fig. 1e shows that counts rapidly diminish during photoexcitation of Dye-FISH labeled cells, reducing by $\sim 12 \%$ in $30 \mathrm{~s}$ and to nearly zero counts in $10 \mathrm{~min}$. This is significant because tens of seconds are needed to acquire a full z-stack for $3 \mathrm{D}$ cell imaging. In comparison, QDs exhibit long-term stability with no significant change in GAPDH mRNA counts $(p>0.05$; Student's $t$-test) after $12 \mathrm{~min}$ of excitation, which is consistent with previous results for QD-based stains measured by net intensity ${ }^{16}$. QD-FISH yielded a significantly higher 3D count number compared with Dye-FISH (by 15-30\%) for cells with the same 2D counts at a single nuclear focal plane (Fig. 1f). This result likely derives from the rapid decline in dye signal, and is the origin of the lower measured labeling density per transcript for dyes compared with $\mathrm{QD}_{13.3}$ (Fig. 1d), which could likely be improved with specialized antifade media and dyes optimized for photostability.

Spot counting fidelity. The ability to identify puncta corresponding to individual molecules through automated algorithms is also significantly improved with QDs compared with dyes. Numerous image analysis algorithms have been developed to recognize individual fluorescently labeled molecules as diffraction-limited spots, each of which invariably applies a hypothesis test to decide whether a spot should be categorized as positive or negative, typically corresponding to a signal-to-noise threshold for a fit to a two-dimensional Gaussian function. The imposed threshold usually requires ad hoc empirical adjustments through human intervention ${ }^{8}$. Spot counting using a scanning window method with serial image depletion (Multiple Target Tracking algorithm ${ }^{17}$ ) is shown in Fig. $2 \mathrm{a}, \mathrm{b}$ for Dye-FISH and QD-FISH, respectively, both using the same probe sequences. The $x$-axis of each plot shows the threshold imposed for spot detection based on the statistical fit of each image spot to a point spread function (described further in Methods). The slope of each positive count curve is plotted in panel c. Compared with DyeFISH, the curve is much flatter for QD-FISH, indicating a lower sensitivity to threshold selection that is critical for robust 
a
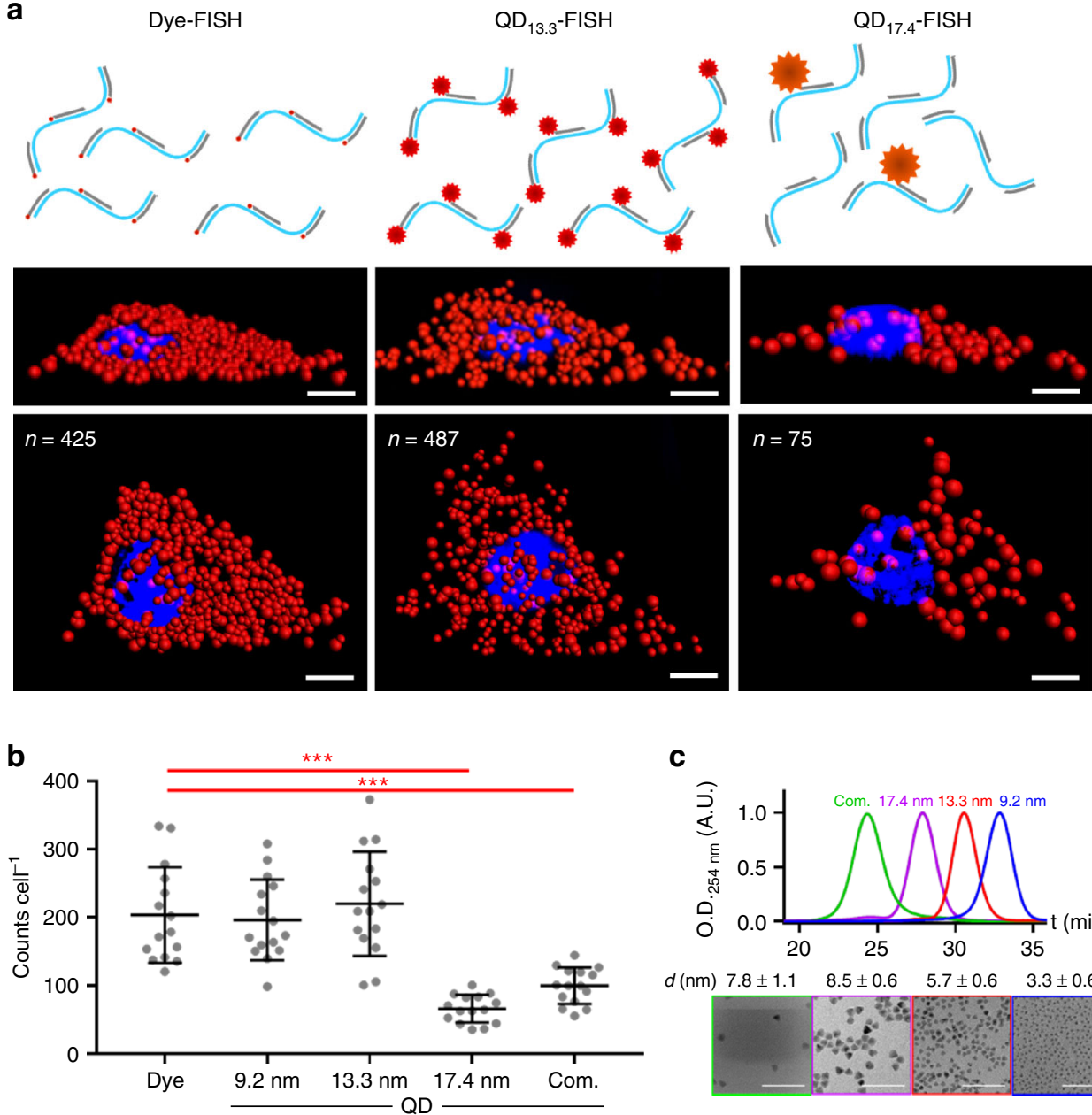

C

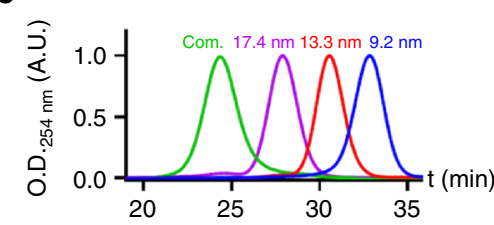

$d(\mathrm{~nm}) 7.8 \pm 1.1 \quad 8.5 \pm 0.6 \quad 5.7 \pm 0.6 \quad 3.3 \pm 0.6$

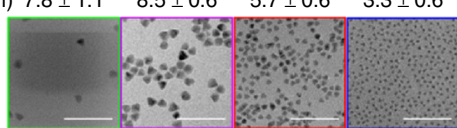

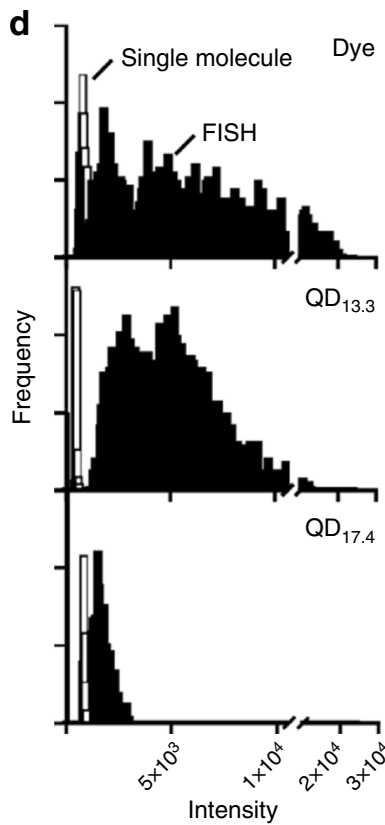

f

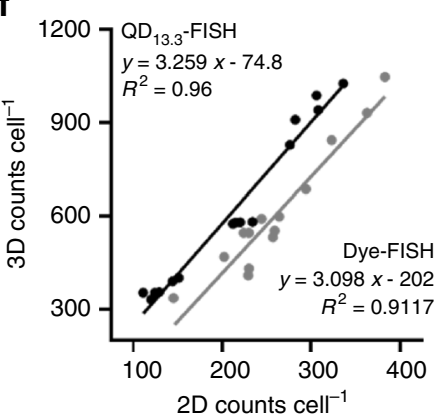

e

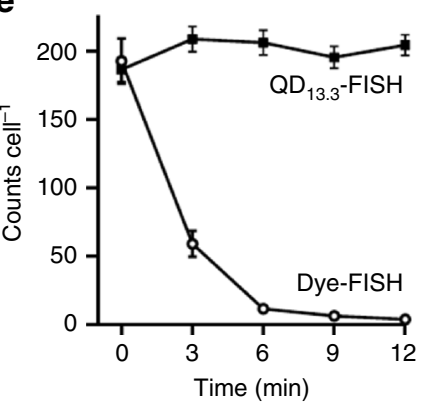

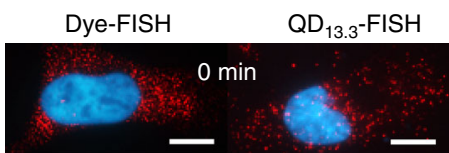

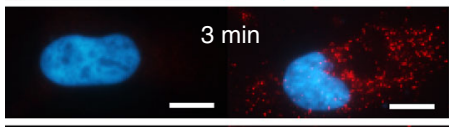

$12 \mathrm{~min}$ g

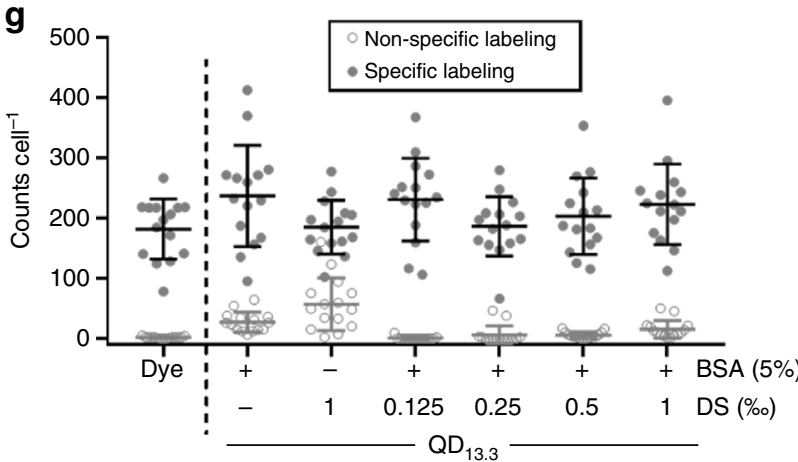

Fig. 1 Fluorescence in situ hybridization (FISH) using dye labels or quantum dot (QD) labels with diverse sizes. Data show HeLa cells stained for glyceraldehyde-3-phosphate dehydrogenase (GAPDH) transcripts. a Schematics show RNA target labeling density; representative 3D deconvolved epifluorescence images show cells in two orthogonal orientations, using dyes, small QDs $(13.3 \mathrm{~nm})$, or big QDs $(17.4 \mathrm{~nm})$. Scale bar $=8 \mu \mathrm{m}$. $\mathbf{b}$ FISH transcript counts (2D) using dyes, custom designed QDs with three hydrodynamic diameters (9.2, 13.3, or 17.4 nm), or commercially available QDs (com.). Asterisks indicate: $p \leq 0.05\left(^{\star}\right), p \leq 0.01\left(^{\star \star}\right)$, and $p \leq 0.001\left(^{\star \star \star}\right)$; Student's $t$-test. $N=15$. Comprehensive statistical comparisons are provided in Supplementary Table 2. c Gel permeation chromatograms and TEM images (with core size) of the four QDs from panel b. Scale bar $=50 \mathrm{~nm}$. $\mathbf{d}$ Intensity histograms of FISH spots for dyes, small QDs $(13.3 \mathrm{~nm})$, and big QDs $(17.4 \mathrm{~nm})$ are shown in black compared with histograms of single-fluorophore intensities in white. e FISH counts after different times of laser excitation, comparing stability of $\mathrm{QD}_{13.3}$ and dyes, including representative images. Scale bars $=10 \mu \mathrm{m} . N=15$. f Correlation between FISH counts in 2D and 3D images for $\mathrm{QD}_{13.3}$ and dye labels. Comprehensive statistical comparisons are provided in Supplementary Table 1. $\mathbf{g}$ Impact of customized blocking conditions on specific and nonspecific labeling. Nonspecific labeling counts (2D) for QDs were statistically the same as those of background when applying both $5 \%$ bovine serum albumin (BSA) and $0.125 \%$ o dextran sulfate (DS). N = 15 . All error bars represent s.d 
a

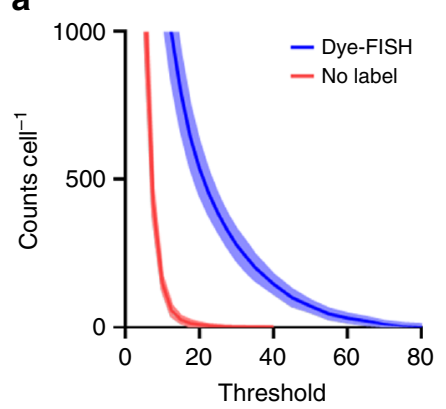

d

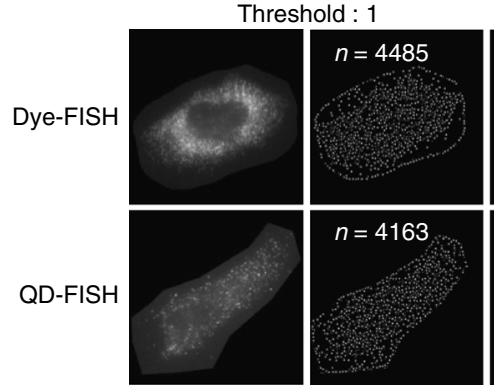

b

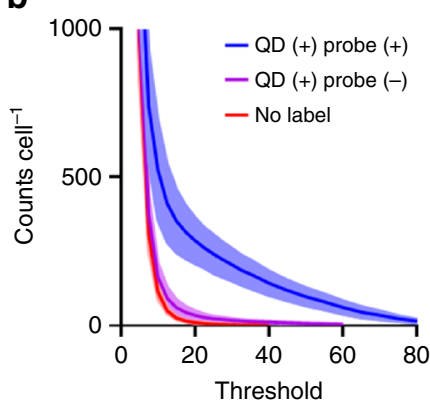

C
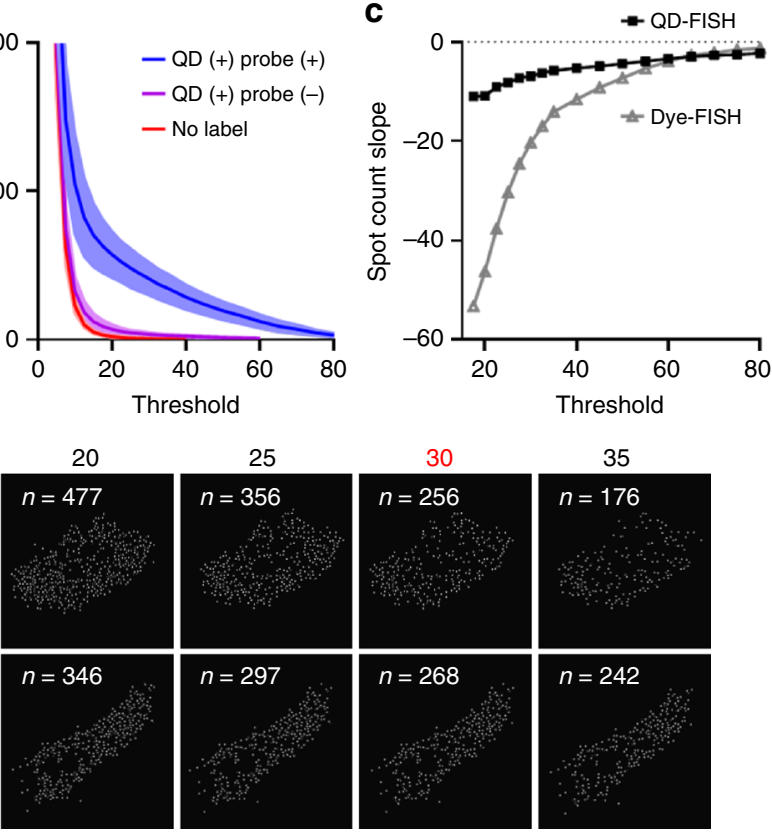

Fig. 2 Computational identification of mRNA spots. a Spot counts in individual cells using Dye-FISH (blue) or cells without labels (red) for different spot detection thresholds (described further in Methods). Shading indicates s.d. of counts between cells. $N=7$. b Spot counts in individual cells using QD-FISH (blue), cells with QDs added but no probe oligonucleotides (purple), or cells without labels (red), for different detection thresholds. Shading indicates s.d. of counts between cells. $N=7$. c Slopes of positive counts plotted against detection threshold. $\mathbf{d}$ Representative 2D images of Dye-FISH and QD-FISH are shown on the left, next to calculated images showing the locations of detected spots in white for each of the threshold values indicated above the images. The higher stability of detection for QD-FISH is evident from the similar numbers of detected spots for each of the threshold values spanning 25-35, compared with a wider range of detected spots for Dye-FISH. Inset numbers, $n$, indicate the number of detected spots

automation to eliminate manual selection biases. This outcome derives from the higher brightness of QDs that is far above the spatially variable autofluorescence background (Fig. 2d), whereas the dye channel is highly convolved with autofluorescence and yields a widely varying spot brightness (Fig. 1d).

In the preceding work, we used a 2-step labeling approach in which QDs attached to streptavidin (SAv) label biotinylated nucleic acid probes pre-hybridized to RNA targets. This was necessary for direct comparison of QD with different sizes, as current QD-nucleic acid conjugates have size-dependent valencies. However, the results are statistically the same when comparing this 2-step labeling process using $\mathrm{QD}_{13.3}$ with 1-step direct labeling in which the same QDs are conjugated with oligonucleotides before addition to cells (Supplementary Fig. 23 ). The 2-step process also allowed identification of specialized blocking conditions to eliminate nonspecific binding of QDs, which, as solid-phase colloids, have a propensity to adsorb to cellular structures. We used an iterative optimization process (Fig. 1g and Supplementary Fig.s 4-6) to find that both bovine serum albumin (BSA) and polyanions (dextran sulfate; DS) reduce nonspecific binding, and their combination has an additive effect, virtually eliminating nonspecific binding when used together. We attribute the success of this blocking cocktail to the elimination of both denatured hydrophobic domains of proteins (by BSA) and polycationic sites (by DS), both of which can adsorb QDs. However QDs could not be mixed directly with DS due to colloidal aggregation, necessitating sequential blocking. Notably DS requires precise concentration control, having diminishing effect at concentrations greater than $0.125 \%$.

Validation of labeling accuracy. To measure the extent to which exact levels of mRNA can be measured in single cells with QDFISH, we modulated transcript numbers in cultured cells using short interfering RNA (siRNA). We focus on the transcript of tumor suppressor phosphatase and tensin homolog (PTEN), a key tumor suppressor gene often deleted in prostate cancer in association with a poor prognosis ${ }^{18,19}$. Representative images for benign prostate hyperplasia (BPH-1) epithelial cells are shown in Fig. 3a, for which an average $75 \%$ reduction in PTEN RNA counts was measured after siRNA treatment (Fig. 3b), a magnitude similar to that measured at the population level by quantitative reverse transcriptase polymerase chain reaction (qRT-PCR) (Fig. 3c). We performed the same analysis in $\mathrm{VCaP}$ prostate cancer cells (Fig. 3d), which are notably much smaller than BPH1 cells. We again observed a similar magnitude of siRNAinduced transcript knockdown through QD-FISH (Fig. 3e) as that observed at the population level with qRT-PCR (Fig. 3f). This outcome is important because mRNA FISH results are challenging to correlate across cell types with different sizes due to differing degrees of spatial overlap of fluorescent spots $^{20}$.

Multiplexed QD-FISH. Finally, we validated the ability to use QDs for multiplexed quantification of multiple mRNA sequences at the single-molecule level. We synthesized three QDs (QD608, QD693, and QD800) with spectrally distinct emission bands (Fig. 4a) by tuning the composition of the $\mathrm{Hg}_{x} \mathrm{Cd}_{1-x} \mathrm{Se}$ alloy domain, which has little impact on QD size but a substantial impact on electronic bandgap ${ }^{21}$. The three QDs were compact and similar in hydrodynamic size after coating with multidentate polymers by GPC (Supplementary Fig. 7). Each QD was conjugated to oligonucleotides complementary to either GAPDH (QD608), PTEN (QD693), or A20 (QD800) mRNA and were then mixed and applied simultaneously to LNCaP prostate cancer cells using the 1-step QD-FISH protocol. Expression levels were modulated by treating the cells with either anti-PTEN siRNA to knock down PTEN expression, or tumor necrosis factor alpha 
a
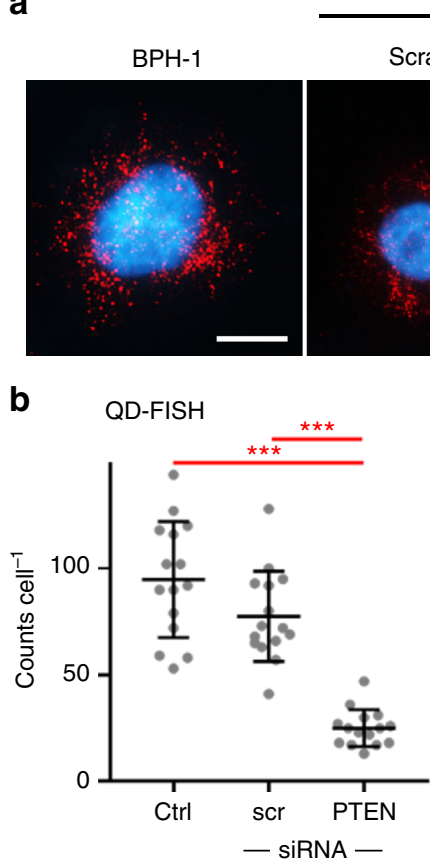

SiRNA

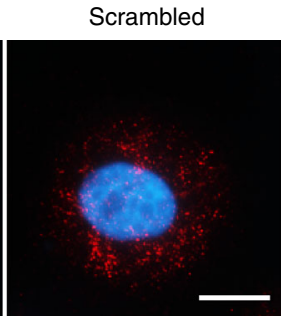

C $\quad$ qRT-PCR

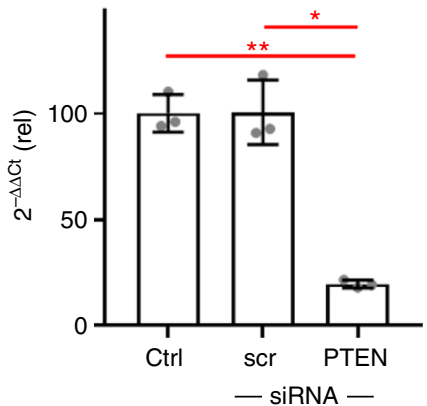

d

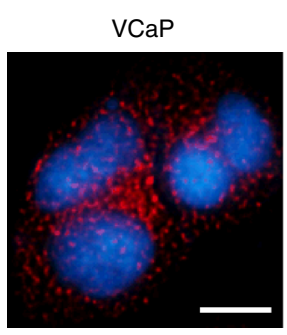

e

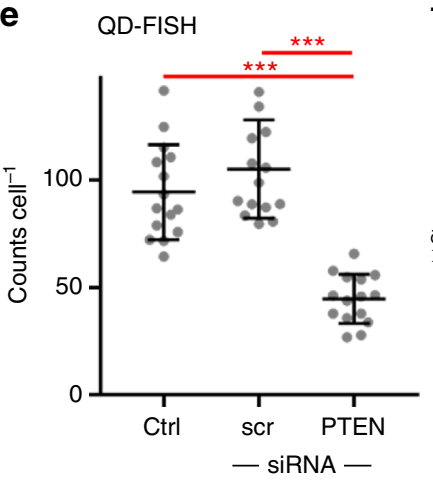

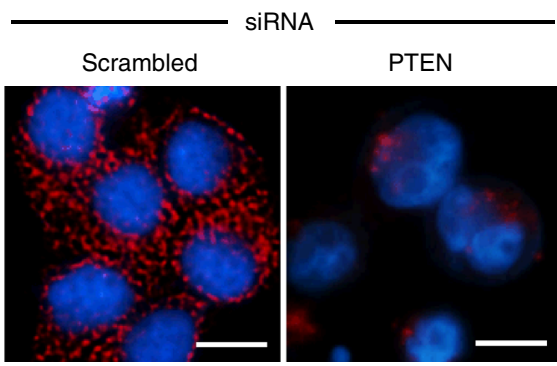

f qRT-PCR

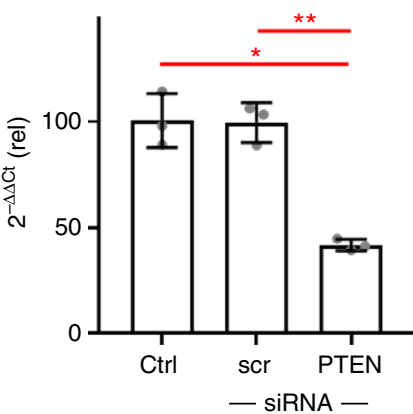

Fig. 3 QD-FISH analysis of phosphatase and tensin homolog (PTEN) transcripts in prostate cancer cell lines. Representative images show BPH-1 cells (a) and VCap cells (d) with or without treatment by siRNA to knock down PTEN expression, or using a scrambled siRNA sequence. Scale bar $=10 \mu \mathrm{m}$. Single-cell QD-FISH counts are shown for (b) BPH-1 cells and (e) VCaP cells, in comparison with transcript measurements by population qRT-PCR for (c) BPH-1 cells and (f) VCap cells. Significantly reduced mRNA levels are observed for anti-PTEN siRNA treatment, with a similar magnitude between QD-FISH and qRTPCR. Asterisks indicate: $p \leq 0.05\left(^{\star}\right), p \leq 0.01\left(^{(\star}\right)$, and $p \leq 0.001\left(^{\star \star \star}\right)$; Student's $t$-test. All error bars represent s.d. $N=15$ for $(\mathbf{b})$ and $(\mathbf{e}), N=3$ for $(\mathbf{c})$ and (f). Comprehensive statistical comparisons are provided in Supplementary Tables 4-7. PTEN mRNA FISH probe sequences are shown in Supplementary Table 8
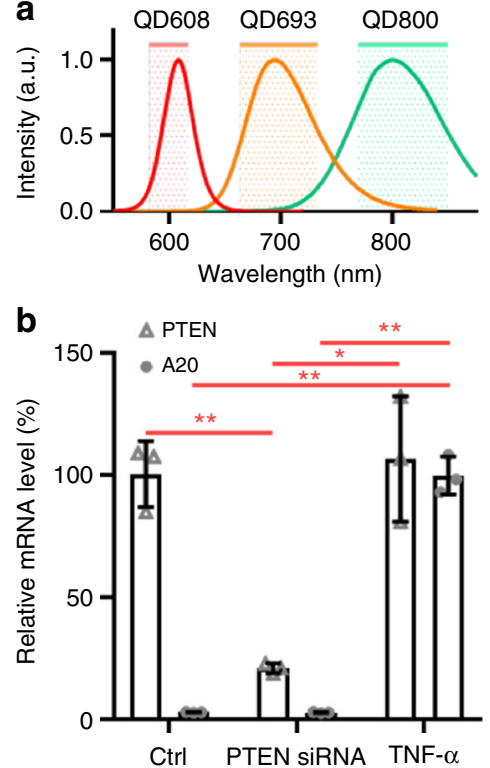

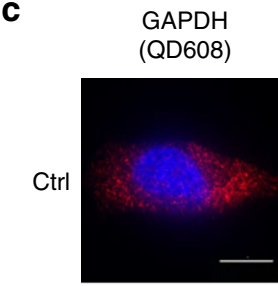

PTEN

(QD693)
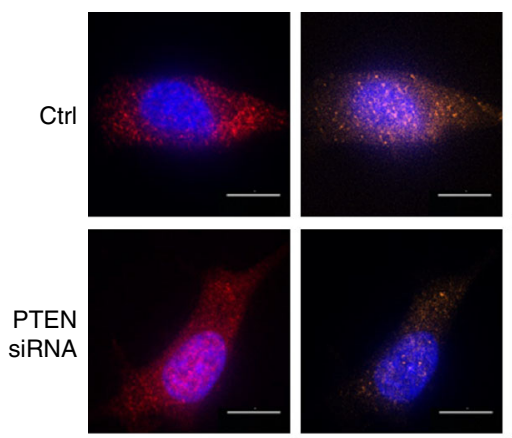

TNF- $\alpha$

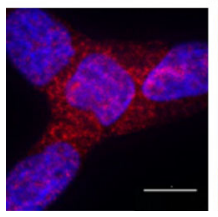

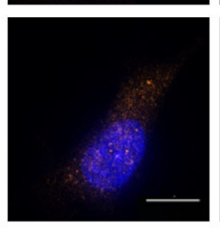

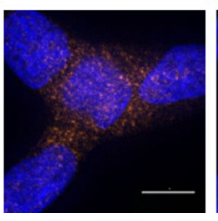

A20 (QD800)
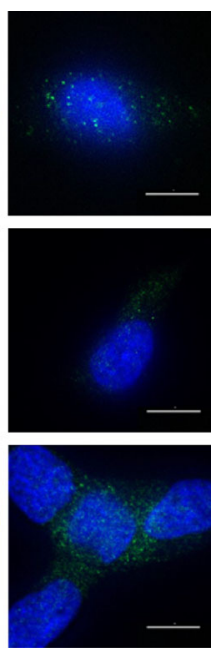

d

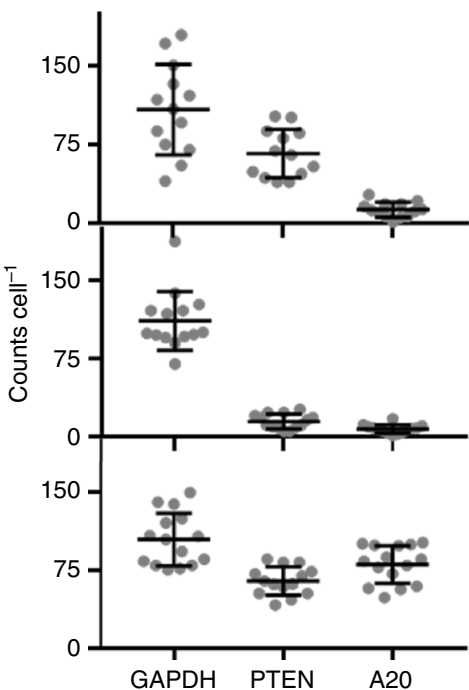

Fig. 4 Multiplexed QD-FISH quantification of three transcripts in single LNCaP cells. The three transcripts include GAPDH, PTEN, and A2O. a Emission spectra of QD608, QD693, and QD800 and corresponding emission bandpass filters used for imaging. b Graph shows the expressions of PTEN and A20 transcripts relative to GAPDH by qRT-PCR after cells were exposed to either anti-PTEN siRNA to knock down PTEN expression or TNF- $\alpha$ to induce A20 expression. Asterisks indicate: $p \leq 0.05\left(^{\star}\right), p \leq 0.01\left(^{(\star}\right)$, and $p \leq 0.001\left(^{(\star \star}\right)$; Student's $t$-test. $N=3$. c Representative QD-FISH images of single cells in each color channel corresponding to bandpass filters shown in panel a. QD probes were specific against GAPDH (QD608), PTEN (QD693) or A20 (QD800), and cells were treated with or without anti-PTEN siRNA or TNF- $\alpha$. Scale bar $=10 \mu \mathrm{m}$. $\mathbf{d}$ Single-cell QD-FISH results are shown for GAPDH, PTEN and $A 20$ transcripts for each of the three experimental conditions corresponding to the images in panel c. $N=15$. All error bars represent s.d. A20 mRNA FISH probe sequences are shown in Supplementary Table 9 
(TNF- $\alpha$ ) to selectively induce $A 20$ gene expression ${ }^{22}$, each validated by qRT-PCR in Fig. 4b. A representative image from each QD-FISH color channel is shown for individual cells in each experimental group in Fig. 4c, and corresponding transcript counts are shown in panel d. By QD-FISH, GAPDH levels were similar in all three experimental groups, whereas PTEN transcript level decreased by $\sim 80 \%$ with anti-PTEN siRNA treatment, and A20 expression significantly increased with TNF- $\alpha$ treatment. These single-cell results correlated well with the population-level qRT-PCR results.

\section{Discussion}

In this work, we identified a critical size threshold limiting the accuracy of RNA labeling in cells and showed that new QDs with compact sizes can label mRNA targets to yield similar counts as those measured with dyes. The ability to tune QD crystalline size independently from fluorescence emission is a key capability of these materials that allowed us to directly measure the impact of probe size on bioanalytical performance without interference from substantial photophysical mismatch. We anticipate that QD-FISH will drastically improve single-molecule FISH studies in thick samples for which repetitive excitation leads to rapid signal deterioration and when autofluorescence limits the accuracy of single-molecule identification. These materials should be well suited for studies requiring high-level multiplexing, as the multispectral tunability of QDs is greater any other current fluorescent probe and can exhibit high efficiency emission in the first and second near-infrared spectra where cellular autofluorescence is negligible and detectors have now become affordable 23,24 . New QD engineering approaches have recently become available to widely tune emission wavelengths without changing the size, which is necessary to maintain total minimum dimensions for accurate labeling ${ }^{14}$. The same physicochemical design rules can further be extended to nanoparticle labels such as rare-earth up-conversion materials and responsive plasmonic materials compatible with unique imaging modalities.

\section{Methods}

Reagents. All chemical reagents were purchased from Sigma-Aldrich or Alfa Aesar unless otherwise specified. QD $\mathrm{D}_{\text {com }}$ was purchased from Thermo-Fisher Scientific as Qdot $^{\text {Ts }} 605$ Streptavidin Conjugate.

QD9.2 synthesis. $\mathrm{QD}_{9.2}$ with emission at $645 \mathrm{~nm}$ and $3.3 \mathrm{~nm}$ diameter by electron microscopy was synthesized with a core/shell $\mathrm{HgCdSe} / \mathrm{CdZnS}$ structure using methods similar to those previously reported ${ }^{14}$. CdSe cores $(2.3 \mathrm{~nm})$ were synthesized using a high-temperature injection reaction between cadmium oxide $(0.6$ $\mathrm{mmol})$, diphenylphosphine selenide $(0.2 \mathrm{mmol})$, and trioctylphosphine selenide (3 $\mathrm{mmol})$ in tetradecylphosphonic acid $(1.33 \mathrm{mmol})$, hexadecylamine $(7.1 \mathrm{~g})$, trioctylphosphine $(7 \mathrm{~mL})$, and 1 -octadecene (ODE; $27.6 \mathrm{~mL}$ ) at $300^{\circ} \mathrm{C}$ for $30 \mathrm{~s}$. After purification, cadmium was partially exchanged with mercury using mercury octanethiolate in oleylamine to yield a $\mathrm{HgCdSe}$ core. After purification, a 2.2monolayer shell of $\mathrm{CdZnS}$ was deposited layer-by-layer in 0.8 -monolayer increments using cadmium acetate in oleylamine $(0.1 \mathrm{M})$, zinc acetate in oleylamine $(0.1 \mathrm{M})$, and elemental sulfur in $\operatorname{ODE}(0.1 \mathrm{M})$ as shell stock solutions. The shell composition comprised 0.8 monolayers of $\mathrm{Cd}_{0.5} \mathrm{Zn}_{0.5} \mathrm{~S}, 1.2$ monolayers of $\mathrm{Cd}_{0.2} \mathrm{Zn}_{0.8} \mathrm{~S}$, and 0.2 monolayers of $\mathrm{ZnS}$.

QD $_{13.3}$ synthesis. $\mathrm{QD}_{13.3}$ with emission at $605 \mathrm{~nm}$ and $5.7 \mathrm{~nm}$ diameter by elec tron microscopy was synthesized with a core/shell CdSe/CdZnS structure using methods similar to those previously reported ${ }^{14}$. CdSe cores $(3.2 \mathrm{~nm})$ were synthesized using a heat-up reaction between cadmium behenate $(1 \mathrm{mmol})$, selenium dioxide $(1 \mathrm{mmol})$, and 1,2 -hexadecanediol $(1 \mathrm{mmol})$ in $\mathrm{ODE}(20 \mathrm{~mL})$ at $230^{\circ} \mathrm{C}$ for $15 \mathrm{~min}$. After purification, a 4.7-monolayer shell of $\mathrm{CdZnS}$ was deposited using the same methodology as that for $\mathrm{QD}_{9.2}$. The shell composition comprised 2.4 monolayers of CdS, 0.8 monolayers of $\mathrm{Cd}_{0.8} \mathrm{Zn}_{0.2} \mathrm{~S}$, and 1.5 monolayers of $\mathrm{ZnS}$.

QD $_{17.4}$ synthesis. $\mathrm{QD}_{17.4}$ with emission at $680 \mathrm{~nm}$ and $8.7 \mathrm{~nm}$ diameter by electron microscopy was synthesized with a core/shell CdSe/CdZnS structure. A CdSe core with a first exciton peak at $645 \mathrm{~nm}$ was synthesized using a method similar to that for $\mathrm{QD}_{13.3}$ with the substitution of cadmium behenate for cadmium myristate.
The shell growth process was similar to that used for $\mathrm{QD}_{9.2}$ to yield a shell composition of 4.0 monolayers of $\mathrm{Cd}_{0.5} \mathrm{Zn}_{0.5} \mathrm{~S}$ and 1.6 monolayers of $\mathrm{ZnS}$.

QD800 synthesis. CdSe cores with emission at $549 \mathrm{~nm}$ were synthesized in a heat-up reaction mixture of cadmium behenate $(0.2 \mathrm{mmol})$, selenium dioxide $(0.2$ $\mathrm{mmol})$, and 1,2 -hexadecanediol $(0.2 \mathrm{mmol})$ in ODE $(5 \mathrm{~mL})$ at $240^{\circ} \mathrm{C}$ for $60 \mathrm{~min}$. After purification, cadmium was partially exchanged with mercury to yield a HgCdSe core by mixing the CdSe cores with mercury acetate in oleylamine and chloroform, followed by the addition of octanethiol to quench the reaction. After purification, a shell of $\mathrm{CdZnS}$ was deposited using the same methodology as that for $\mathrm{QD}_{9.2}$ to yield a shell composition of 2.4 monolayers of $\mathrm{CdS}, 0.8$ monolayers of $\mathrm{Cd}_{0.5} \mathrm{Zn}_{0.5} \mathrm{~S}$, and 0.8 monolayers of $\mathrm{ZnS}$.

QD693 synthesis. The synthesis was the same as that used for QD800, except the CdSe core emission wavelength maximum was $558 \mathrm{~nm}$ and mercury exchange was performed with mercury octanethiolate in oleylamine to reduce the degree of redshift.

QD coating and conjugation. $\mathrm{QD}_{9.2}, \mathrm{QD}_{13.3}$, and $\mathrm{QD}_{17.4}$ were coated with polyacrylamido(histamine-co-TEG-co-azido-TEG) (P-IM-N $\left.\mathrm{N}_{3}\right)$ to generate aqueous azide-functional colloids ${ }^{13}$. Dibenzocyclooctyne (DBCO)-functionalized streptavidin (SAv) was prepared by mixing SAv (AnaSpec) in phosphate buffered saline (PBS; $0.5 \mathrm{mg} \mathrm{mL}^{-1}$ ) with a solution of DBCO- $N$-hydroxysuccinimidyl ester (DBCO-NHS, Click Chemistry Tools) in DMSO $(2.5 \mathrm{mM})$ at a molar ratio of 1:5, followed by repeated pipetting and incubation on ice for $2 \mathrm{~h}$. The conjugate was purified by centrifugal filtration using a filter with $3 \mathrm{kDa}$ molecular weight cutoff (MWCO) at $4^{\circ} \mathrm{C}$. Azide-functional QDs in PBS were then mixed 1:1 with DBCO$\mathrm{SAv}$ (and other ratios for optimization) and allowed to react at room temperature overnight. The reaction was quenched by adding a 50-fold molar excess of 2azidoacetic acid on ice for $15 \mathrm{~min}$. These conjugates were used directly for 2-step QD-FISH. For 1-step QD-FISH, QD-SAv conjugates were mixed with biotinlabeled probes at a 1:1 molar ratio for $1 \mathrm{~h}$ at room temperature.

QD characterization. Absorption spectra of QD dispersions were acquired using an Agilent Cary 5000 UV-Vis-NIR spectrophotometer. Fluorescence spectra of QD dispersions were collected using a Horiba NanoLog spectrofluorometer, with solutions diluted to eliminate self-quenching. Signal acquisition conditions such as scan time, slit widths, and number of scans were adjusted so that the brightest sample was not saturating the detector (photomultiplier tube) and such that all spectra showed sufficiently high signal-to-noise ratios to yield smooth curves. Transmission electron microscopy images of QDs were obtained using a JEOL 2010 LaB6 high-resolution microscope in the Frederick Seitz Materials Research Laboratory Central Research Facilities at the University of Illinois. Samples were prepared by placing a drop of dilute QD solution in hexane or chloroform on an ultrathin carbon film TEM grid (Ted Pella, \#01824) and then wicking the solution off with a tissue. QD-SAv conjugates were characterized by agarose gel electrophoresis (Supplementary Fig. 8) using excess biotin-labeled DNA to confirm SAv conjugation to QDs by a migration shift ${ }^{13}$. The DNA sequence was 5 -Biotin/(T)68 TAGCCA GTG TAT CGC AAT GAC G-3' (Integrated DNA Technologies). QDSAv was incubated with biotin-DNA at room temperature for $15 \mathrm{~min}$ and electrophoresis was performed in a $2 \%$ polyacrylamide, $0.5 \%$ agarose gel at $4{ }^{\circ} \mathrm{C}$.

Cells. HeLa cells (ATCC) were cultured in Eagle's Minimum Essential Medium (EMEM) with $10 \%$ fetal bovine serum (FBS) and $1 \%$ penicillin/streptomycin (P/S) at $37^{\circ} \mathrm{C}$ in $5 \% \mathrm{CO}_{2}$. BPH-1 and LNCaP (ATCC) cells were cultured in RPMI Medium 1640 with $10 \%$ FBS and $1 \% \mathrm{P} / \mathrm{S}$ at $37^{\circ} \mathrm{C}$ in $5 \% \mathrm{CO}_{2}$. VCaP (ATCC) cells were cultured in Dulbecco's Modified Eagle's Medium (DMEM) with 10\% FBS and $1 \% \mathrm{P} / \mathrm{S}$ at $37^{\circ} \mathrm{C}$ in $5 \% \mathrm{CO}_{2}$. For FISH studies, cells $\left(1 \times 10^{5}\right)$ were seeded on $18 \mathrm{~mm}$ round \#1 coverglass in each well of a 12 -well cell culture plate and cultured until $70 \%$ confluent. The cells were then washed with PBS, fixed with $4 \%$ paraformaldehyde for $10 \mathrm{~min}$ at room temperature, and permeabilized with $70 \%(\mathrm{v} / \mathrm{v})$ ethanol for $24 \mathrm{~h}$ at $4{ }^{\circ} \mathrm{C}$. For qRT-PCR analysis, BPH-1, VCaP and LNCaP cells $\left(3 \times 10^{5}\right)$ were seeded in 6 -well plates and cultured until $80 \%$ confluent. For PTEN mRNA silencing, cells were transfected with anti-PTEN or scrambled siRNA (Santa Cruz) using Lipofectamine 2000 (Thermo Fisher Scientific) following manufacturer protocols at a siRNA concentration of $1.5 \mathrm{fM}$ for $24 \mathrm{~h}$. The medium was then aspirated and the cells were washed with PBS and fixed in $4 \%$ paraformaldehyde in PBS at room temperature for $10 \mathrm{~min}$. After two washes with PBS, the cells were permeabilized with $70 \%(\mathrm{v} / \mathrm{v})$ ethanol for at least $1 \mathrm{~h}$ at $2{ }^{\circ} \mathrm{C}$ to $8{ }^{\circ} \mathrm{C}$.

Nucleic acid probes. GAPDH mRNA Dye-FISH nucleic acid probes were synthesized and optimized by LGC Biosearch Technologies, labeled with either biotin or CAL Fluor ${ }^{\circledast}$ Red 590 Dye. Probes targeting human PTEN mRNA (NM_000314.6) and A20 mRNA (NM_001270508.1) were designed using Stellaris ${ }^{\circledR}$ Probe Designer (version 4.2, LGC Biosearch Technologies) and are provided in Supplementary Tables 8 and 9 . 
Dye-FISH. GAPDH mRNA Dye-FISH was performed following standard procedures $^{8}$ using Wash Buffers A and B and Hybridization Buffer supplied by the probe manufacturer. Probe incubation was performed for $16 \mathrm{~h}$ in the dark at $37^{\circ} \mathrm{C}$, nuclei were stained with Hoechst 33342 (Thermo Fisher Scientific), and each coverglass was mounted on a slide with $90 \%$ glycerol in PBS, sealed using nail polish.

Two-step QD-FISH. Biotin-labeled FISH probes with the same sequences used for Dye-FISH (LGC Biosearch Technologies) were hybridized with fixed and permeabilized cells on coverglass using identical protocols for Dye-FISH and nuclei were stained with Hoechst 33342. The cells were then blocked for $2 \mathrm{~h}$ with blocking conditions as indicated. The optimized mixture contained BSA and DS in $2 x$ saline-sodium citrate (SSC) buffer at $\mathrm{pH}$ 7.2. After aspirating the blocking buffer, cells were incubated with $10 \mathrm{nM}$ QD-SAv in $1 \%$ (w/v) BSA in $2 \times$ SSC buffer at room temperature for $2 \mathrm{~h}$. The ratio between QD:SAv and the time of incubation were independently optimized (Supplementary Fig. 9 and 10). Cells on coverglass were then washed three times with Wash Buffer B before mounting on slides with $90 \%$ glycerol in PBS, sealed using nail polish.

One-step QD-FISH. Fixed and permeabilized cells on coverglass were washed with Wash Buffer A for $5 \mathrm{~min}$ and incubated with Hybridization Buffer for $30 \mathrm{~min}$, followed by the optimized blocking buffer from 2-step QD-FISH for $2 \mathrm{~h}$. The cells were then incubated in a mixture of biotin-probe conjugates of QD-SAv ( $8 \mathrm{nM}$ QD) in $10 \%$ formamide, $0.33 \mathrm{mg} \mathrm{m}^{-1}$ yeast RNA, $10 \mathrm{mM}$ ribonucleoside vanadyl complex, $0.1 \%$ BSA, and $2 \mathrm{XSSC}$ for $16 \mathrm{~h}$ in the dark at $37^{\circ} \mathrm{C}$ in a sealed humidified chamber. The coverglass was then washed with Wash Buffer A at $37^{\circ} \mathrm{C}$ for 30 min and nuclei were stained with Hoechst 33342 for 30 min. The coverglass was then washed with Wash Buffer B for 5 min before mounting on slides with $90 \%$ glycerol in PBS, sealed with nail polish.

Multiplexed QD-FISH. LNCaP cells were transfected with anti-PTEN siRNA as described above. A20 gene expression was induced by treatment with TNF- $\alpha$ (100 $\mathrm{ng} / \mathrm{mL}$ ) in complete medium for $1 \mathrm{~h}$ at $37^{\circ} \mathrm{C}$. The cells were then processed following the 1-step QD-FISH procedure and incubated in a mixture of the three QD$\mathrm{SAv}$ conjugates, each conjugated to biotinylated oligonucleotides complementary to mRNA sequences of GAPDH, PTEN, or A20. QD-FISH signals of QD608, QD693 and QD800 were collected using $488 \mathrm{~nm}$ laser excitation and 600/37 nm, 698/70 $\mathrm{nm}$ or $809 / 81 \mathrm{~nm}$ bandpass emission filters, respectively.

Imaging. Immediately after preparation, cells were imaged on a Zeiss Axio Observer Z1 inverted microscope with an EC Plan-Neofluar $100 \times 1.45$ N.A. oilimmersion objective. Images were collected with a Photometrics eXcelon Evolve 512 EMCCD camera controlled through Zeiss Zen software. Hoechst was imaged using $100 \mathrm{~W}$ halogen lamp excitation with a $365 \mathrm{~nm}$ excitation filter and 445/50 nm emission filter; CAL Fluor ${ }^{\circledR}$ Red 590 Dye was imaged using $561 \mathrm{~nm}$ laser excitation and a $600 / 37 \mathrm{~nm}$ bandpass emission filter. $\mathrm{QD}_{13.3}$ and commercial QDs were imaged using $488 \mathrm{~nm}$ laser excitation and a $600 / 37 \mathrm{~nm}$ bandpass emission filter. $\mathrm{QD}_{17.4}$ and $\mathrm{QD}_{9.2}$ were imaged using $488 \mathrm{~nm}$ laser excitation and a $585 \mathrm{~nm}$ longpass emission filter. Z-stack images of entire cells were collected in $0.22 \mu \mathrm{m}$ increments. For each sample, 20 areas on the coverglass were selected at random for imaging. To obtain single-molecule fluorescence intensity values, dye-probes and QD-SAv conjugates dispersed in PBS were adsorbed on glass coverslips and imaged via epifluorescence microscopy using identical conditions to those used for FISH images. For each sample, videos during continuous excitation were acquired to identify single molecules by their distinct intensity time-traces using MATLAB algorithms ${ }^{14}$.

Image analysis. Images were exported as 8-bit uncompressed TIFF files. For 2D image analysis, spot counting in individual cells was performed using the Multiple Target Tracking (MTT) Algorithm based in MATLAB ${ }^{17}$ to determine the location and intensity of each spot. For 3D z-stacks, files were deconvolved using AUTOQUANT X3 (Media Cybernetics, Inc.) and analyzed using IMARIS (Bitplane) for $3 \mathrm{D}$ distribution reconstruction and signal spot counting. To ensure that deconvolution did not alter spot numbers, MTT analysis of 2D images was performed before and after deconvolution (Supplementary Fig. 11). In the MTT algorithm, spot detection in images is performed by evaluating each $7 \times 7$ window in the image using a generalized likelihood ratio test to decide if a spot fits a point spread function, assuming Gaussian noise. Thus the analysis accounts for local background values, rather than global intensities, which is beneficial to account for the nonuniformity of autofluorescence across a cell. The algorithm also subtracts each spot and repeats the analysis until all spots are detected, which is beneficial when a high spatial density of spots is present. The threshold applied for the hypothesis test is normalized as the probability of false positives per $512 \times 512$ image (false positives per $\sim 250,000$ windows). In Fig. 2, the indicated threshold is the probability of false positive of detected spots in logarithmic scaling, in units of decibels.

QRT-PCR. Total RNA from cells in 6 well plates was extracted using a RNeasy Mini Kit (QIAGEN) and reverse-transcribed using a High-Capacity cDNA Reverse Transcription Kit (Thermo Fisher Scientific). Primer sequences for human GAPDH were 5'-AGG GCT GCT TTT AAC TCT GGT-3' and 5'-CCC CAC TTG ATT TTG GAG GGA-3'. Primer sequences for human PTEN were 5'-CAA GAT GAT GTT TGA AAC TAT TCC AAT G-3' and 5'-CCT TTA GCT GGC AGA CCA CAA-3'. Primer sequences for human A20 were 5'-GAC CAT GGC ACA ACT CAT CTC A-3' and 5'-GTT AGC TTC ATC CAA CTT TGC GGC ATT G-3'25-28. All primers were obtained from Integrated DNA Technologies. Real-time qPCR was performed on a Mastercycler ${ }^{\circledR}$ RealPlex2 (Eppendorf).

Statistical analysis. Data are presented as mean \pm s.d. Statistical significance was determined using Student's $t$-test and analysis of variance (one-way ANOVA) using GraphPad Instat 3 software. After comparing the overall difference between groups, the Tukey's honestly significant difference (HSD) post-hoc test was used to specify where the differences occurred between groups.

\section{Data availability}

The data that support the findings of this study are available from the corresponding author upon reasonable request.

Received: 27 April 2018 Accepted: 21 September 2018 Published online: 26 October 2018

\section{References}

1. Gall, J. G. \& Pardue, M. L. Formation and detection of RNA-DNA hybrid molecules in cytological preparations. Proc. Natl. Acad. Sci. USA 63, 378-383 (1969).

2. John, H. A., Birnstiel, M. L. \& Jones, K. W. RNA-DNA hybrids at the cytological level. Nature 223, 582-587 (1969).

3. Langer-Safer, P. R., Levine, M. \& Ward, D. C. Immunological method for mapping genes on Drosophila polytene chromosomes. Proc. Natl. Acad. Sci. USA 79, 4381-4385 (1982).

4. Levsky, J. M. \& Singer, R. H. Fluorescence in situ hybridization: past, present and future. J. Cell. Sci. 116, 2833-2838 (2003).

5. Femino, A. M., Fay, F. S., Fogarty, K. \& Singer, R. H. Visualization of single RNA transcripts in situ. Science 280, 585-590 (1998).

6. Crosetto, N., Bienko, M. \& van Oudenaarden, A. Spatially resolved transcriptomics and beyond. Nat. Rev. Genet. 16, 57-66 (2015).

7. Buxbaum, A. R., Haimovich, G. \& Singer, R. H. In the right place at the right time: visualizing and understanding mRNA localization. Nat. Rev. Mol. Cell Biol. 16, 95-109 (2015)

8. Raj, A., van den Bogaard, P., Rifkin, S. A., van Oudenaarden, A. \& Tyagi, S Imaging individual mRNA molecules using multiple singly labeled probes. Nat. Methods 5, 877-879 (2008).

9. Lubeck, E., Coskun, A. F., Zhiyentayev, T., Ahmad, M. \& Cai, L. Single-cell in situ RNA profiling by sequential hybridization. Nat. Methods 11, 360-361 (2014).

10. Kairdolf, B. A. et al. Semiconductor quantum dots for bioimaging and biodiagnostic applications. Ann. Rev. Anal. Chem. 6, 143-162 (2013).

11. Ioannou, D. et al. Quantum dots as new-generation fluorochromes for FISH: an appraisal. Chromosome Res. 17, 519-530 (2009).

12. Ioannou, D. \& Griffin, D. K. Nanotechnology and molecular cytogenetics: the future has not yet arrived. Nano Rev. 1, 5117-5130 (2010).

13. Ma, L. et al. Multidentate polymer coatings for compact and homogeneous quantum dots with efficient bioconjugation. J. Am. Chem. Soc. 138, 3382-3394 (2016).

14. Lim, S. J. et al. Brightness-equalized quantum dots. Nat. Comm. 6, 8210-8219 (2015).

15. Baum, M., Erdel, F., Wachsmuth, M. \& Rippe, K. Retrieving the intracellular topology from multi-scale protein mobility mapping in living cells. Nat. Comm. 5, 4494-4505 (2014).

16. Wang, L. et al. Single molecule localization imaging of telomeres and centromeres using fluorescence in situ hybridization and semiconductor quantum dots. Nanotechnology 29, 285602-285610 (2018).

17. Serge, A., Bertaux, N., Rigneault, H. \& Marguet, D. Dynamic multiple-target tracing to probe spatiotemporal cartography of cell membranes. Nat. Methods 5, 687-694 (2008)

18. Kwabi-Addo, B. et al. Haploinsufficiency of the PTEN tumor suppressor gene promotes prostate cancer progression. Proc. Natl. Acad. Sci. USA $\mathbf{9 8}$ 11563-11568 (2001)

19. Murphy, S. J. et al. Integrated analysis of the genomic instability of PTEN in clinically insignificant and significant prostate cancer. Mod. Pathol. 29 143-156 (2016)

20. Coskun, A. F. \& Cai, L. Dense transcript profiling in single cells by image correlation decoding. Nat. Methods 13, 657-660 (2016) 
21. Smith, A. M. \& Nie, S. Bright and compact alloyed quantum dots with broadly tunable near-infrared absorption and fluorescence spectra through mercury cation exchange. J. Am. Chem. Soc. 133, 24-26 (2011).

22. Golovko, O., Nazarova, N. \& Tuohimaa, P. A20 gene expression is regulated by TNF, Vitamin D and androgen in prostate cancer cells. J. Steroid Biochem. Mol. Biol. 94, 197-202 (2005).

23. Smith, A. M., Mancini, M. C. \& Nie, S. M. Second window for in vivo imaging. Nat. Nanotech. 4, 710-711 (2009).

24. Bruns, O. T. et al. Next-generation in vivo optical imaging with short-wave infrared quantum dots. Nat. Biomed. Engr. 1, 0056-0066 (2017).

25. Shen, Y. H. et al. Up-regulation of PTEN (phosphatase and tensin homolog deleted on chromosome ten) mediates p38 MAPK stress signal-induced inhibition of insulin signaling. A cross-talk between stress signaling and insulin signaling in resistin-treated human endothelial cells. J. Biol. Chem. 281, 7727-7736 (2006).

26. Kim, S. et al. Down-regulation of the tumor suppressor PTEN by the tumor necrosis factor-alpha/nuclear factor-kappaB (NF-kappaB)-inducing kinase/ NF-kappaB pathway is linked to a default IkappaB-alpha autoregulatory loop. J. Biol. Chem. 279, 4285-4291 (2004).

27. Gupta, A. \& Dey, C. S. PTEN, a widely known negative regulator of insulin/ PI3K signaling, positively regulates neuronal insulin resistance. Mol. Biol. Cell. 23, 3882-3898 (2012).

28. Gunzl, P. et al. Anti-inflammatory properties of the PI3K pathway are mediated by IL-10/DUSP regulation. J. Leukoc. Biol. 88, 1259-1269 (2010).

\section{Acknowledgements}

This work was supported by funds from the Mayo-Illinois Alliance, the National Institutes of Health (R33CA196458 to J.C.C., S.J.M., F.K., and A.M.S.) and the National Science Foundation I-Corps Program (1745812 to A.M.S.). P.L. was supported by the National Institute of Biomedical Imaging and Bioengineering of the National Institutes of Health under Award Number T32EB019944 and the National Science Foundation Grant 0965918 IGERT: Training the Next Generation of Researchers in Cellular and Molecular Mechanics and BioNanotechnology. L.M. was supported by the 2017-2018 UIUC Graduate College Dissertation Completion Fellowship. S.J.L. acknowledges support from the Start-up Fund and Basic Research Programme (18-NT-01) of DGIST funded by the Ministry of Science and ICT of Korea.

\section{Author Contributions}

Y.L. performed quantum dot conjugation, cell studies, and microscopy. L.M. and Z.H. performed quantum dot polymer coating and chromatographic analysis. Y.L. and P.L. performed image and data analysis. S.J.L. and S.S. prepared quantum dot cores and performed electron microscopy. A.M.S., Y.L., S.J.M., F.K., G.V., and J.C.C. designed and supervised the experiments. A.M.S. and Y.L. wrote the manuscript.

\section{Additional information}

Supplementary Information accompanies this paper at https://doi.org/10.1038/s41467018-06740-x.

Competing interests: The authors declare no competing interests.

Reprints and permission information is available online at http://npg.nature.com/ reprintsandpermissions/

Publisher's note: Springer Nature remains neutral with regard to jurisdictional claims in published maps and institutional affiliations.

(c) (1) Open Access This article is licensed under a Creative Commons Attribution 4.0 International License, which permits use, sharing, adaptation, distribution and reproduction in any medium or format, as long as you give appropriate credit to the original author(s) and the source, provide a link to the Creative Commons license, and indicate if changes were made. The images or other third party material in this article are included in the article's Creative Commons license, unless indicated otherwise in a credit line to the material. If material is not included in the article's Creative Commons license and your intended use is not permitted by statutory regulation or exceeds the permitted use, you will need to obtain permission directly from the copyright holder. To view a copy of this license, visit http://creativecommons.org/ licenses/by/4.0/.

(C) The Author(s) 2018 